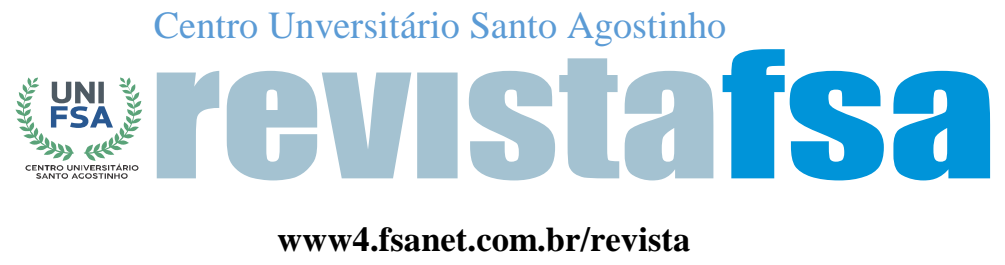

Rev. FSA, Teresina, v. 18, n. 7, art. 4, p. 55-76, jul. 2021

e-revist@s http://dx.doi.org/10.12819/2021.18.7.4

\title{
Internet das Coisas Aplicada aos Negócios: Análise das Publicações Utilizando o Bibliometrix
}

Internet of Things Applied to Business: Analysis of Publications Using Bibliometrix

\section{Anderson Betti Frare}

Doutorado em Contabilidade pela Universidade Federal de São Carlos Mestre em Contabilidade pela Universidade Federal do Rio Grande anderson_betti_frare@hotmail.com

Alex Sandro Rodrigues Martins

Mestre em Contabilidade pela Universidade Federal do Rio Grande alexmartins@furg.br

Carla Milena Gonçalves Fernandes

Mestra em Contabilidade pela Universidade Federal do Rio Grande carlafernandes@furg.br Vagner Horz

Mestre em Contabilidade pela Universidade Federal do Rio Grande vagnerhorz@gmail.com

Alexandre Costa Quintana

Doutor em Controladoria e Contabilidade pela Universidade de São Paulo professorquintana@hotmail.com

Endereço: Anderson Betti Frare

(UFSC), Campus Universitário Reitor João David Ferreira Lima, s/no ${ }^{\circ}$ Trindade - Florianópolis - SC CEP: 88040-900. Brasil.

Endereço: Alex Sandro Rodrigues Martins (FURG) Avenida Itália, Km. 8, s/n, bairro Carreiros, CEP: 96201.900, Rio Grande - RS. Brasil.

Endereço: Carla Milena Gonçalves Fernandes (FURG) Avenida Itália, Km. 8, s/n, bairro Carreiros, CEP: 96201.900, Rio Grande - RS. Brasil.

Endereço: Vagner Horz

(FURG) Avenida Itália, Km. 8, s/n, bairro Carreiros, CEP: 96201.900, Rio Grande - RS. Brasil.

Endereço: Alexandre Costa Quintana

(FURG) Avenida Itália, Km. 8, s/n, bairro Carreiros, CEP: 96201.900, Rio Grande - RS. Brasil.
Editor-Chefe: Dr. Tonny Kerley de Alencar Rodrigues

Artigo recebido em 07/06/2021. Última versão recebida em 27/06/2021. Aprovado em 28/06/2021.

Avaliado pelo sistema Triple Review: a) Desk Review pelo Editor-Chefe; e b) Double Blind Review (avaliação cega por dois avaliadores da área).

Revisão: Gramatical, Normativa e de Formatação 


\title{
RESUMO
}

Objetiva-se analisar as publicações sobre Internet of Things (IoT) no universo dos negócios, presentes na base da Web of Science. O portfólio de 2.632 documentos fora analisado no package Bibliometrix no software $\mathrm{R}$. O maior número de artigos concentra-se no IEEE ACCESS The Multidisciplinary Open Access Journal. Acerca dos autores e universidades mais prolíferas, denota-se a predominância no continente asiático. Em relação aos artigos mais citados e às palavras-chave, evidencia-se a conexão entre os temas tecnológicos (automação, sistemas e controle) e a facilitação na gestão para as respectivas operações.

Palavras-chave: Internet das Coisas. IoT. Negócios. Bibliometrix.

\begin{abstract}
The aim is to analyze the publications about Internet of Things (IoT) in the business universe, present at the base of the Web of Science. The portfolio of 2,632 documents was analyzed in the Bibliometrix package in the $\mathrm{R}$ software. The largest number of papers is focused on the IEEE ACCESS The Multidisciplinary Open Access Journal. Among the most prolific authors and universities, there is a predominance in Asia. In relation to the most cited articles and the keywords, the connection between the technological themes (automation, systems and control) and the facilitation in the management of the respective operations.
\end{abstract}

Keywords: Internet of Things. IoT. Business. Bibliometrix. 


\section{INTRODUÇÃO}

Internet das Coisas, Internet of Things ou IoT: Três denominações comuns para um objeto de mesmo sentido. $\mathrm{O}$ conceito para tais termos expressa-se em uma tecnologia disruptiva, capaz de conectar qualquer coisa ou pessoa em qualquer momento e local, por meio de quaisquer redes ou serviços (BALDINI, 2018; LU; PAPAGIANNIDIS; ALAMANOS, 2018; MAN; NA; KIT, 2015).

O termo "things" ou "coisas" não recebe tal denominação em vão, sendo literalmente compreensível, uma vez que a tecnologia IoT pode ser aplicada aos mais diversos campos, como no vestuário, alimentação, equipamentos, ferramentas, materiais, trabalhos artísticos e negócios (KOSMATOS; TSELIKAS; BOUCOUVALAS, 2011). Em particular no contexto dos negócios, as organizações estão tendo significativos impactos (LU et al., 2018; SHIN, 2014; STANKOVIC, 2014).

Neste ínterim, Galegale et al. (2016) realizaram uma bibliometria sobre a IoT aplicada ao mundo dos negócios, utilizando-se da base da Web of Science, no período de 1992 a 2013. Constataram poucos estudos com este foco, sendo a maioria aplicada a outras áreas. De maneira geral, os autores concluíram que existe um amplo campo de pesquisa da IoT no contexto de gestão e negócios.

Similarmente, Lu et al. (2018) revisaram a literatura de IoT no campo de negócios, por meio das bases de dados Scopus, Ebsco e Web of Science, analisando todo período existente até fevereiro de 2017, resultando em 91 artigos. Os autores utilizaram de diversos filtros, como áreas de ciências sociais, artes e humanidades, gestão de negócios, contabilidade, psicologia ou áreas multidisciplinares.

Para um panorama geral da IoT, Mishra et al. (2016) realizaram uma revisão da literatura nas bases Scopus e Web of Science, no período de 2000 a 2015, analisando diversas variáveis. Erfanmanesh e Abrizah (2018) estenderam a análise descrita na assertiva anterior para o período de 2011 a 2016, mediante a base de dados da Scopus. Ambas as publicações evidenciaram um drástico aumento de publicações sobre IoT a partir de 2014, denotando a contemporaneidade do tema.

Devido a esse exponencial crescimento de publicações acerca do tema IoT nos últimos anos (ERFANMANESH; ABRIZAH, 2018), principalmente de 2014 para cá, assim como a existência de um grande campo de pesquisa do IoT no contexto dos negócios, fora evidenciada com base nas publicações até 2013 (GALEGALE et al., 2016). Levando-se em conta a ampliação dos filtros utilizados por Lu et al. (2018), surge a seguinte problemática de 
pesquisa: Quais são as características das publicações científicas da IoT no mundo dos negócios?

Desta maneira, o objetivo permeia a análise das publicações sobre a IoT no universo dos negócios, na base da Web of Science. Mediante a ampliação dos filtros de áreas considerados por Lu et al. (2018), como finanças, pesquisa operacional, métodos quantitativos aplicados às ciências sociais, administração pública e outras, pretende-se, assim, estender a contextualização de "negócios" em consonância à IoT.

A justificativa para a pesquisa permeia as transformações que a IoT vem causando, inclusive nos usuários. Um novo paradigma tecnológico está sendo construído, sendo capaz de conectar objetos físicos e virtuais mediante a internet, independentemente do momento ou lugar. Não obstante, o mundo dos negócios encontra-se imerso nessas mudanças propiciadas pela IoT (BALDINI et al., 2018; DIAS, 2016; GALEGAGE et al., 2016; LU et al., 2018; MAN et al., 2015; OBERLÄNDER et al., 2018; VAN DEURSEN; MOSSBERGER, 2018; YANG; YANG; PLOTNICK, 2013).

O presente artigo contribui ao expandir o período de análise do estudo de Galegale et al. (2016) e a contextualização de negócios (LU et al., 2018), auxiliando na evidenciação das tendências mundiais da IoT no amplo contexto da gestão e dos negócios. Ademais, tornam-se possíveis comparações com o mapeamento da literatura geral de IoT, elucidada nos estudos de Erfanmanesh e Abrizah (2018) e Mishra et al. (2016).

O artigo também assume o viés educativo, no contexto de exemplificar a utilização do package bibliometrix no software $\mathrm{R}$, o qual facilita drasticamente o processo de análise de produções científicas (ARIA; CUCCURULLO, 2017). Evidencia-se que até o momento da construção da presente investigação, não se encontrou pesquisa alguma no Brasil que tivesse utilizado tal package na área de negócios.

A estrutura do artigo compõe-se em cinco seções. Essa primeira introduz o tema e denota sua pertinência. Na sequência, expõe-se a revisão da literatura, expondo os conceitos de IoT bem como sua aplicação no mundo dos negócios. A terceira seção explicita os procedimentos metodológicos adotados para a realização de tal investigação, seguindo para a análise dos dados encontrados e, por último, as considerações finais. 


\section{REVISÃO DA LITERATURA}

A literatura foi revisada em dois momentos. Inicialmente, os conceitos, aplicabilidades e discussões do IoT no contexto multidisciplinar. Após, na segunda subseção, aborda-se exclusivamente a aplicação desta no universo dos negócios.

\subsection{IoT - Conceitos e aplicações}

A possibilidade de interconexão entre pessoas em distintos lugares a qualquer momento de modo virtual torna-se cada vez mais possível, sendo este movimento de conectividade proporcionado por um novo paradigma intitulado Internet das Coisas (BONOMI et al., 2012; BOTTA et al., 2016; LEE; LEE, 2015; LU et al., 2018; ZANELLA et al., 2014).

À medida que esse mundo inteligente é construído por meio de sensores, dispositivos e aparatos tecnológicos mais sofisticados, os quais viabilizem interatividade entre indivíduos e coisas, há uma propensão para que essa sinergia esteja voltada a novos sistemas que possibilitem a formação e desenvolvimento de serviços (STANKOVIC, 2014). Assim, o termo Internet das Coisas surge em uma complexa gama de aplicações virtuais a partir do impulsionamento de governos que buscam, por exemplo, aprimorar o uso desses mecanismos na gestão pública (ZANELLA et al., 2014).

Por conseguinte, a articulação que permite essa evolução de transferência e interação de informações dá-se de forma ainda inicial, em virtude da necessidade premente de uma sofisticação tanto no processamento quanto na análise de dados. Além disso, também ocorrem preocupações quanto à confiabilidade e autonomia para que as informações circulantes nas redes sem fio estejam protegidas e atuem de forma dinâmica quando da ocorrência de possíveis problemas (GUBBI et al., 2013).

Nesse sentido, Botta et al. (2016, p. 684) corroboram expondo que a Internet das Coisas "representa uma das tecnologias mais disruptivas, permitindo cenários de computação onipresentes e abrangentes", bem como busca propiciar que novos avanços vinculados à tecnologia da comunicação articulem-se a fim de conduzir uma convergência de dados e informações disponíveis, hoje, na internet. Para tanto, vislumbra-se que essa dinâmica de conhecimento promova meios de melhoramento em áreas como, por exemplo, de produção e custos de empresas (LEE; LEE, 2015). 
Diante o exposto, adiciona-se ainda que um dos desafios inerente ao assunto Internet das Coisas circunda o alto custo despendido pelas empresas ao investirem em tal tecnologia (LEE; LEE, 2015). Para tanto, o caráter inovador presente na IoT possibilita que seu aprimoramento e aplicação estejam alicerçados por segurança bem como políticas de privacidade. Além disso, dentre algumas de suas vantagens, principalmente de cunho organizacional, encontram-se perspectivas de melhores monitoramentos e controles dos serviços (SICARI et al., 2015).

Em sequência, destaca-se que um dos fatores norteadores da existência da IoT consiste na interação da tecnologia e comunicação em virtude de a concatenação destes subsidiarem melhores condições de monitoramento, rastreamento e identificação de diferentes atividades no intuito de facilitar a operacionalização dessa tecnologia (IoT) em ambientes organizacionais. Dessa maneira, outra vantagem atribuída à IoT faz menção à possibilidade de inserção de programas únicos capazes de propiciarem múltiplas interações entre os usuários, inseridos na organização ou fora dela, no cumprimento dos objetivos estabelecidos (ATZORI; LERA; MORABITO, 2010).

Em consonância, a tecnologia Internet das Coisas torna-se relevante devido sua aplicabilidade em organizações que têm como objetivo o estabelecimento de metas, as quais permitam uma composição significativa de serviços, suportes e análises. Ademais, o fluxo heterogêneo de informações torna-se mais viável, pois os sistemas são capazes de produzirem respostas mais rápidas (BONOMI et al., 2012).

Para Erfanmanesh e Abrizah (2018), a IoT representa a incorporação de várias tecnologias por meio de ferramentas tecnológicas inteligentes que podem fomentar condições de mudanças em diversos segmentos, como: área econômica, da saúde, da política, assim como na esfera ambiental. Além disso, Man, Na e Kit (2015) expõem que a IoT emergiu como uma revolução tecnológica que teria como incumbência ampliar os horizontes relacionados à comunicação e fazer com que áreas como a de logística e negócios industriais prosperassem, aumentando assim o valor econômico das vendas e por conseguinte das transações realizadas.

Em um contínuo, inclui-se que a partir da otimização dos serviços proporcionada pela IoT conduz a ações viabilizadas entre as pessoas dentro de uma organização, como: estímulo à criação e desenvolvimento de novos serviços, além do aumento da transparência entre as partes interessadas (ZANELLA et al., 2014). Aliás, apoiando-se nessas ações, a Internet das Coisas constitui-se em uma ferramenta tecnológica capaz de produzir soluções satisfatórias em universos organizacionais competitivos (SICARI et al., 2015). 


\subsection{IoT aplicada aos negócios}

Um dos principais aspectos que envolvem a IoT consiste no impacto e no efeito de seu uso em cenários como: saúde, segurança, negócios, etc. (BOTTA et al., 2016). Assim, no que concerne à área de negócios, a tecnologia Internet das Coisas e os estudos até o momento realizados, ainda se encontram incipientes nas pesquisas que relacionam essa tecnologia disruptiva no âmbito dos negócios (LU et al., 2018). Do ponto de vista econômico e de gestão, esse conceito foi primeiramente cunhado por Elgar Fleisch em 2010. O conceito de IoT não é novo, mas só recentemente tornou-se relevante do ponto de vista prático nos negócios, em razão dos avanços no desenvolvimento de hardware, especialmente na última década (FLEISCH, 2010).

Nesse aspecto, um dos valores intrínsecos a essa ampliação tecnológica diz respeito à expectativa de progressão na área de negócios organizacionais por meio da Internet das Coisas, em virtude de considerar que este aparato tecnológico venha a corroborar com o crescimento de forma significativa na área de serviços que envolvam a internet. Salienta-se ainda que a existência de uma ramificação da IoT, aliada à gestão de negócios, propicia um campo cujas mudanças e inovações atuam de forma expressiva, perpassando o campo de pesquisas atingindo transações estratégicas de grandes corporações (XU, 2012).

Para a implementação da IoT nos negócios visa otimizar processos produtivos. Quando as máquinas se comunicam, possibilitam que as interferências das pessoas sejam minimizadas, em processos de fabricação, permitindo que, por meio dos dados coletados, as máquinas sinalizem o momento em que devem ocorrer as manutenções, com análise de dados históricos e uso de técnicas estatísticas proporcionando prevenções de falhas no sistema. Assim, evitando gastos desnecessários, a quebra de aparelhos e, consequentemente, pausas na produção, proporcionando ótimos benefícios para as indústrias e fabricantes (CHEN, 2017).

Para Porter e Heppelmann (2014), os produtos baseados em IoT permitem uma alteração radical nos negócios existentes e já estão estabelecidos há um longo período. Como exemplo, um fabricante de determinado produto - por meio do acesso aos dados do produto e pela habilidade de antecipar, reduzir e reparar falhas - tem uma capacidade sem precedentes de melhorar o desempenho do produto e aprimorar os serviços prestados aos clientes. Nesse novo mundo do negócio, os clientes pagam conforme usam o produto/serviço, diferentemente do modelo atual, no qual os clientes pagam previamente pelo produto/serviço (PORTER; HEPPELMANN, 2014). 
No estudo, Iansiti e Lakhani (2014) analisam vários casos de inovação dos modelos de negócio em empresas que já estão se adaptando à tecnologia digital e à IoT, como a General Electric (GE), a Microsoft, a International Business Machines (IBM), SAP e o Google. Os autores listam lições aprendidas dos casos reais estudados por eles, que podem ser consideradas como facilitadores para as empresas que desejam modelar seus negócios a produtos/serviços baseados em IoT (IANSITI; LAKHANI, 2014).

Desse modo, nos sistemas intercomunicantes, a consequência é o aumento de produtividade, criando novas estratégias de produção e permitindo o melhor conhecimento do mercado. Com a implementação de IoT, métodos tradicionais precisam ser alterados e a resistência por parte de empresas deve ser vencida (CHEN, 2017).

\section{METODOLOGIA}

Consonante a questão formulada, acerca dos objetivos, a pesquisa classifica-se como descritiva, uma vez que procura dissertar sobre o panorama e perspectivas da IoT no contexto dos negócios. Relativo à forma como o problema é abordado, utiliza-se de metodologia quantitativa, por meio de análises e comparações de origem estatísticas e/ou matemáticas (MARTINS; THEÓPHILO, 2009).

Para a escolha do portfólio de publicações, utilizaram-se alguns critérios, dentre eles: (i) base de dados da Web of Science; (ii) possuir o termo: internet of things contidos nos títulos; iii) somente artigos. Foram encontrados 8.102 documentos. A amostra final resultou em 2.632 de acordo com os critérios estabelecidos para este estudo.

Após a filtragem descrita anteriormente, realizou-se o download das informações dos artigos em formato bibtex, para posterior análise no software $\mathrm{R}$. O tratamento destes dados ocorreu com o auxílio do package "Bibliometrix", o qual torna-se interessante para a análise de big data referentes a publicações científicas, facilitando a organização das variáveis, assim como proporcionando análises amplas e complexas de forma sistematizada (ARIA; CUCCURULLO, 2017).

O download do package Bibliometrix pode ser efetuado pelo endereço (http://www.bibliometrix.org/), porém para sua operacionalização torna-se necessário o download do software R (https://cran.r-project.org/) e do Rstudio (https://www.rstudio.com). Ambos são softwares livres e complementares: O Rstudio é uma interface que atua de forma integrada ao R, facilitando sua utilização. 
O package Bibliometrix suporta arquivos no formato bibtex, que podem ser originados das seguintes bases de dados: SCOPUS (http://www.scopus.com); Clarivate Analytics Web of Science (WoS) (http://www.webofknowledge.com); Cochrane Database of Systematic Reviews(http://www.cochranelibrary.com/cochranedatabaseofsystematicreviews/index.html); eRISmedPubMed/MedLine(https://www.ncbi.nlm.nih.gov/pubmed/).

Após a seleção e download do portfólio de artigos, os dados devem ser importados no R e/ou Rstudio para efetuar as análises. Existe a opção de utilizar a função "shiny”, que possibilita a partir dos softwares citados o uso do aplicativo biblioshiny (http://www.bibliometrix.org/biblioshiny.html), mediante interface web.

O bibliometrix pode gerar diversos recursos visuais com base nos dados em análise, como gráficos, figuras e tabelas. Porém, ressalta-se que o pesquisador possui a opção de importar os dados resultantes da análise para outros softwares, em diversos formatos, seja para abordar visual ou expressamente de forma diferente.

\section{ANÁLISE DOS DADOS}

A pesquisa analisou o período de 2002 até o primeiro trimestre de 2019 com intuito de conhecer o panorama sobre a produção científica voltadas à IoT, diante dessa questão foram encontrados 2.686 documentos que versam sobre o tema, sendo 2.632 artigos, um capítulo de livro e 53 artigos que evidenciaram IoT nos procedimentos metodológicos. O quantitativo de publicações ligadas à IoT encontradas nesse estudo, seguem a mesma representatividade evidenciadas nos estudos de Whitmore, Agarwal e Xu (2015); Galegale et al. (2016); Erfanmanesh e Abrizah (2018) e Medeiros et al. (2018).

Porém, algumas dessas obras encontradas na pesquisa têm uma abordagem voltada para Iot na tecnologia, segundo Whitmore, Agarwal e Xu (2015), essa é uma tendência dessa temática, visto que num primeiro momento está ligada especificadamente à tecnologia da informação e com isso necessitando estudos voltados para outras áreas do conhecimento.

Dessa forma, as publicações sobre IoT, está dividida em 638 fontes, as quais são representadas por revistas, livros, entre outros. Diante disso, a pesquisa de Pacheco, Klein e Righi (2016) apresentou que o tema IoT vem ganhando espaço nos mais variados tipos de meios de publicação, essa afirmação pode ser observada nessa pesquisa. No período analisado, observou-se que 6.265 autores dedicaram-se a estudar esse tema, realizando em média 15,77 citações em suas obras, em relação à aparição dos autores nas obras, de forma geral o número passa para 10.043, uma vez que 210 documentos da amostra são referentes 
apenas a um único autor e 6.055 produções científicas estão relacionadas a pesquisas com autores vinculados a mais de uma obra.

Cabe salientar que, em média, cada obra possui 2,33 autores e 3,74 coautores, e esses estudos no período analisado indicaram a utilização de 2.224 vezes a palavra IoT como sendo uma das palavras-chaves para indicar o tema.

Na sequência é apresentada a Figura 1, que proporciona conhecer a evolução das publicações sobre o tema IoT, no período de 2002 até 02 de abril de 2019, em nível internacional.

\section{Figura 1 - Produção Científica Anual}

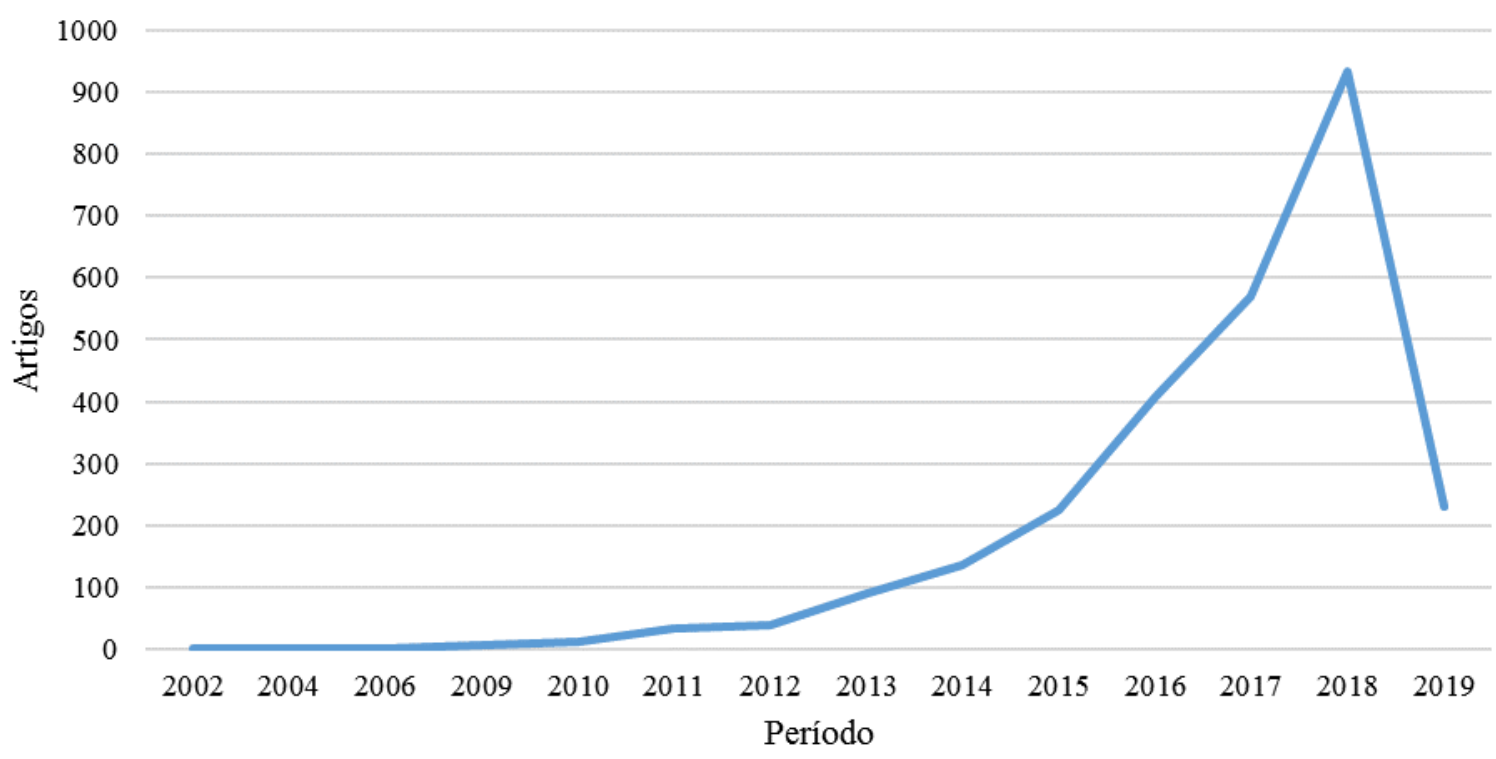

Fonte: Dados da pesquisa.

A Figura 1 retrata que as publicações sobre essa temática vêm num crescente no lapso temporal analisado, outro fator a ser destacado é que a partir de 2009 inicia-se uma evolução significativa de obras voltadas para IoT, visto que nesse ano ocorreram sete publicações sobre IoT, que comparando com o ano anterior representa seis obras a mais publicadas e esse crescimento a cada ano ficou mais expressivo, chegando ao ponto de no ano de 2018 serem publicadas 933 pesquisas voltadas à Internet das Coisas em nível internacional.

Nesse contexto, Erfanmanesh e Abrizah (2018) ressaltaram em seu estudo esse mesmo crescimento nas publicações voltadas para área de tecnologia e especial vinculadas à IoT. Os autores relataram que o número de pesquisas relacionadas à IoT evidenciadas na plataforma de busca Scopus, aumentou quase sete vezes, de 747 em 2011 para 4.989 em 2016. Seguindo a mesma lógica, a pesquisa de Galegale et al. (2016), relatou um aumento expressivo a partir de 2002 nas publicações sobre a IoT, em decorrência de uma valorização do meio acadêmico 
e consequentemente uma maior aceitação dos periódicos acadêmicos para publicações que abordem esse tema.

Neste sentido, observa-se a importância que os estudiosos têm dado ao tema, considerando sua relevância no cotidiano das pessoas e organizações (MAN; NA; KIT, 2015).

Na sequência, a Tabela 1 evidencia as dez fontes de pesquisas mais citadas quando o tema a ser tratado refere-se à Internet das Coisas.

Tabela 1 - Fontes Mais Relevantes

\begin{tabular}{lcc}
\hline Fontes & & Artigos \\
\hline IEEE ACCESS THE MULTIDISCIPLINARY OPEN ACCESS JOURNAL & 187 \\
\hline IEEE INTERNET OF THINGS JOURNAL & 171 \\
\hline SENSORS & & 136 \\
\hline FUTURE GENERATION COMPUTER & SYSTEMS-THE & INTERNATIONAL \\
JOURNAL OF ESCIENCE & 102 \\
\hline INTERNATIONAL JOURNAL OF DISTRIBUTED SENSOR NETWORKS & 83 \\
\hline IEEE COMMUNICATIONS MAGAZINE & 62 \\
\hline IEEE TRANSACTIONS ON INDUSTRIAL INFORMATICS & 46 \\
\hline WIRELESS PERSONAL COMMUNICATIONS & 44 \\
\hline INTERNATIONAL JOURNAL OF ONLINE ENGINEERING & 37 \\
\hline COMPUTER NETWORKS & 31 \\
\hline
\end{tabular}

Fonte: Dados da pesquisa.

Corroborando com os achados de Erfanmanesh e Abrizah (2018), a Tabela 1 buscou demonstrar as fontes por meio dos locais que continham no mínimo 10 artigos que explanassem acerca do tema internet das coisas. Assim, pode-se perceber que o local onde ocorrera o maior número de artigos fora em IEEE ACCESS The Multidisciplinary Open Access Journal, que de igual forma reitera o encontrado por Erfanmanesh e Abrizah (2018). Observa-se um crescimento significativo de publicações sobre o tema no IEEE Internet of Things Journal em oposição ao encontrado no estudo Mishra et al. (2016).

No que tange à proficuidade, mediante a Figura 2, observaram-se os 20 autores com maiores números de publicações acerca da IoT. No eixo x evidencia-se o nome dos autores, enquanto no eixo y expõe-se a quantidade de artigos produzido por cada um. 
Figura 2 - Autores Profícuos

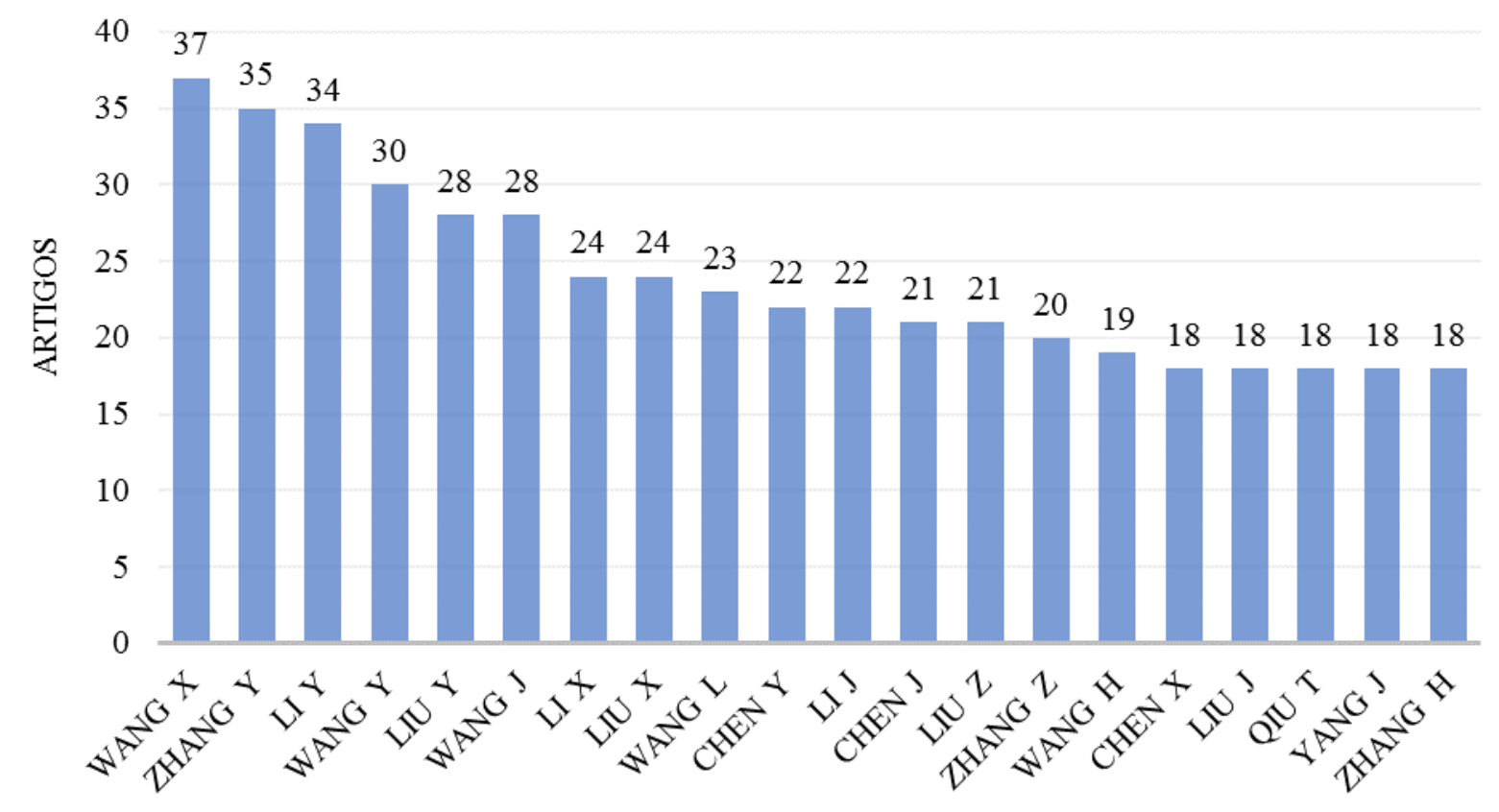

AUTORES

Fonte: Dados da pesquisa.

Denota-se a pertinência do continente asiático nos autores mais profícuos, sendo Wang $\mathrm{X}$ o mais prolífero, com 37 artigos. Além deste, outros três autores possuem 30 ou mais publicações. Estes achados vislumbram uma nova perspectiva, pois no estudo de Erfamanesh e Abrizah (2018) os autores mais produtivos entre 2011 e 2016 eram apenas europeus, americanos ou da Oceania.

Nesta perspectiva de proficuidade dos autores, elucidaram-se os pressupostos da Lei de Lotka, acerca da produção científica em relação à quantidade de autores. Conforme Lotka (1926), o número de autores que produzem contribuições científicas em certo campo do conhecimento aproxima-se de $1 / \mathrm{n}^{2}$ dos que possuem uma única produção, assim como estes que têm uma única produção correspondem a cerca de 60\%. Na Tabela 2 seguem as informações dentre este ínterim da Lei de Lotka. 
Tabela 2 - Lei De Lotka

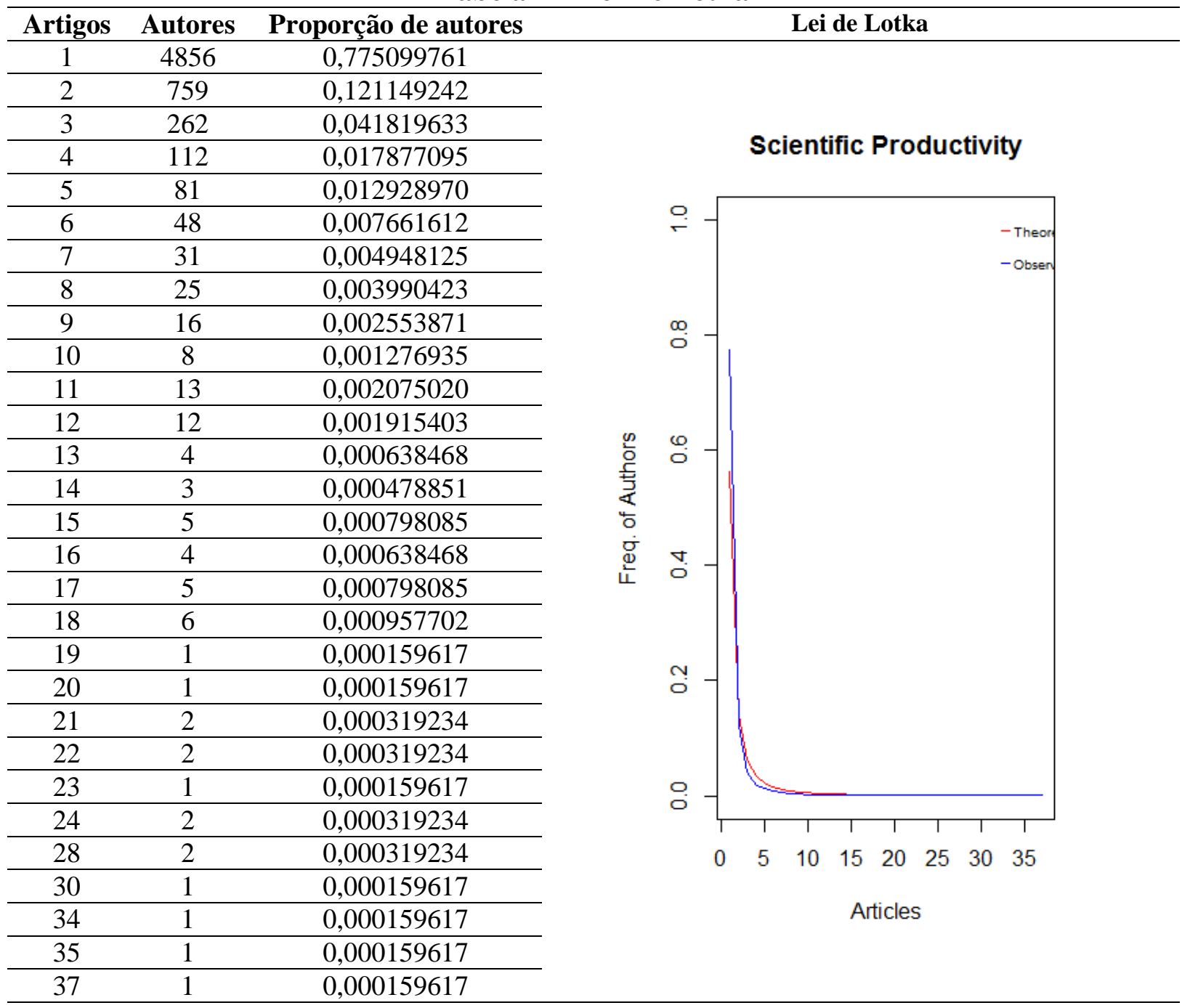

Fonte: Dados da pesquisa.

Mediante a Tabela 2, observa-se que cerca de $77 \%$ dos autores possuem uma única publicação, sendo essa proporção acima do exposto por Lotka (1926). Tal fato pode ser explicado em parte devido ao grande número da amostra, assim como a diversidade de aplicações da IoT e sua exponencial ascensão nas publicações e aparatos tecnológicos, no contexto global (ERFANMANESH; ABRIZAH, 2018; LU et al., 2018).

No que concerne aos pressupostos da Lei de Lotka (1926), estes foram integralmente atendidos, apresentando significância a 0,01, conforme o teste de duas amostras estipulado por Kolmogorov-Smirnoff. Desta maneira, observa-se que a distribuição de autores por artigos segue estreitamente a distribuição evidenciada por Lotka (1926).

Ao perpassar este panorama relativo aos autores, denota-se a relevância de elucidar as afiliações (universidades ou institutos) que originaram os maiores números de publicações em 
relação à amostra obtida. Estas informações juntamente com os países de origem de cada país são expostas na Tabela 3.

Tabela 3 - Afiliações Profícuas

\begin{tabular}{clc}
\hline Artigos & \multicolumn{1}{c}{ Afiliações } & País \\
\hline 83 & Beijing University of Posts and Telecommunications & China \\
57 & King Saud University & Arábia Saúdita \\
39 & Dalian University of Technology & China \\
38 & University of Electronic Science and Technology of & China \\
& China & \\
37 & Nanjing University of Posts and Telecommunications & China \\
35 & Universidade Jiao Tong de Xangai & China \\
33 & Huazhong University of Science and Technology & China \\
29 & Vellore Institute of Technology & Índia \\
28 & Beihang University & China \\
28 & Kyungpook National University & Coréia do Sul \\
28 & University of Science and Technology Beijing & China \\
27 & Northeastern University & Estados Unidos \\
26 & Central South University & China \\
26 & University of Surrey & Reino Unido \\
26 & Xidian University & China \\
24 & Tianjin University & China \\
23 & Aalto University & Finlândia \\
23 & Beijing Jiaotong University & China \\
23 & Southeast University & China \\
23 & Wuhan University & China \\
\hline
\end{tabular}

Fonte: Dados da pesquisa.

Sobre as instituições com maior número de publicações no contexto da IoT, destacamse as instituições situadas na China, sendo que no ranking das 20 mais neste quesito, $70 \%$ são deste país. Neste ínterim, Erfanmanesh e Abrizah (2018) evidenciaram que das 15 universidades com maiores quantidades de artigos com este tema, 60\% eram chinesas. Este achado demonstra o engajamento das universidades e pesquisadores em questão na produção científica de IoT.

Com relação ao número de artigos produzidos por países, a Figura 3 demonstra a predominância do tema IoT em publicações de autores de país único (Single Country Publications - SCP) e publicações de autores de diversos países (Multiple Country Publications - MPC). 
Figura 3 - Países De Procedência Das Publicações

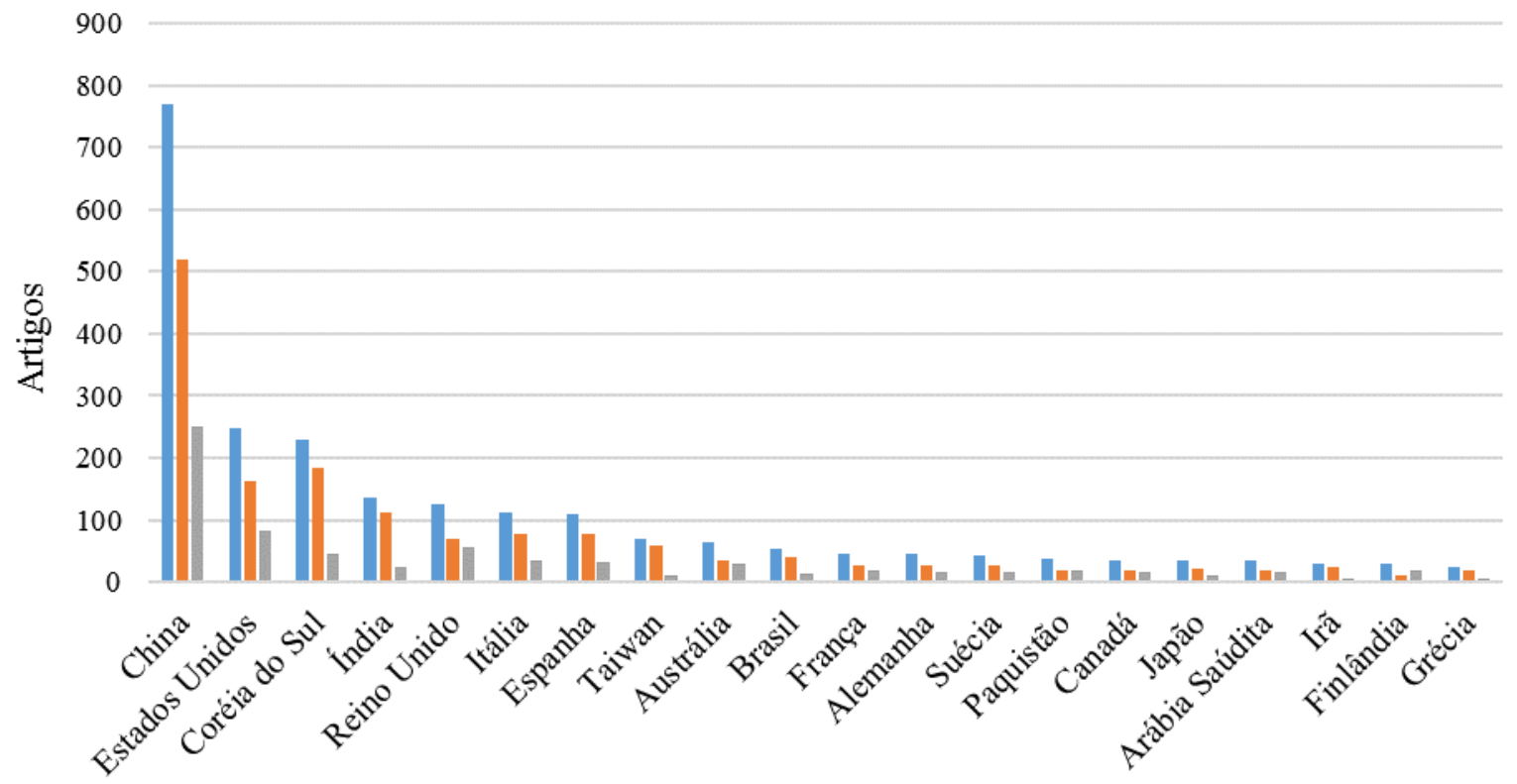

Países

$\square$ Artigos $\square \mathrm{SCP} \square \mathrm{MCP}$

Fonte: Dados da pesquisa.

A partir do exposto pela Figura 3, pode-se perceber a existência do tema IoT inserido em vários países, ou seja, há uma internacionalização das publicações com o passar dos anos. Salienta-se que dentre os 20 países mais representativos, 8 pertencem ao continente asiático, como por exemplo, a China, cuja quantidade de publicações encontradas foi a mais expressiva, corroborando com os achados no estudo de Erfanmanesh e Abrizah (2018).

No que concerne aos artigos mais citados e seus respectivos números de citações, cabe à Tabela 4 demonstrar tais informações.

Tabela 4 - Artigos Mais Citados

\begin{tabular}{|c|c|c|}
\hline $\begin{array}{l}\text { Artigo } \\
\end{array}$ & $\mathbf{N}^{\circ}$ Citações & TC por ano \\
\hline $\begin{array}{l}\text { Atzori, L., Iera, A., \& Morabito, G. (2010). The internet of things: A } \\
\text { survey. Computer networks, 54(15), 2787-2805. }\end{array}$ & 4101 & 455,667 \\
\hline $\begin{array}{l}\text { Gubbi, J., Buyya, R., Marusic, S., \& Palaniswami, M. (2013). } \\
\text { Internet of Things (IoT): A vision, architectural elements, and future } \\
\text { directions. Future generation computer systems, 29(7), 1645-1660. }\end{array}$ & 2585 & 430,833 \\
\hline $\begin{array}{l}\text { Zanella, A., Bui, N., Castellani, A., Vangelista, L., \& Zorzi, M. } \\
\text { (2014). Internet of things for smart cities. IEEE Internet of Things } \\
\text { journal, 1(1), 22-32. }\end{array}$ & 1066 & 213,2 \\
\hline $\begin{array}{l}\text { Al-Fuqaha, A., Guizani, M., Mohammadi, M., Aledhari, M., \& } \\
\text { Ayyash, M. (2015). Internet of things: A survey on enabling } \\
\text { technologies, protocols, and applications. IEEE communications } \\
\text { surveys \& tutorials, 17(4), 2347-2376. }\end{array}$ & 1062 & 265,5 \\
\hline Miorandi, D., Sicari, S., De Pellegrini, F., \& Chlamtac, I. (2012). & 1058 & 151,143 \\
\hline
\end{tabular}


Internet of things: Vision, applications and research challenges. $A d$ hoc networks, 10(7), 1497-1516.

Da Xu, L., He, W., \& Li, S. (2014). Internet of things in industries: A survey. IEEE Transactions on industrial informatics, 10(4), 22332243.

Perera, C., Zaslavsky, A., Christen, P., \& Georgakopoulos, D. (2014). Context aware computing for the internet of things: A survey. IEEE communications surveys \& tutorials, 16(1), 414-454.

Kortuem, G., \& Kawsar, F. (2010, November). Market-based user innovation in the Internet of Things. In 2010 Internet of Things (IOT) (pp. 1-8). IEEE.

Stankovic, J. A. (2014). Research directions for the internet of things. IEEE Internet of Things Journal, 1(1), 3-9.

Botta, A., De Donato, W., Persico, V., \& Pescapé, A. (2016). Integration of cloud computing and internet of things: a survey. Future generation computer systems, 56, 684-700.

Bandyopadhyay, D., \& Sen, J. (2011). Internet of things: Applications and challenges in technology and standardization. Wireless personal communications, 58(1), 49-69.

Islam, S. R., Kwak, D., Kabir, M. H., Hossain, M., \& Kwak, K. S. (2015). The internet of things for health care: a comprehensive survey. IEEE Access, 3, 678-708.

Jin, J., Gubbi, J., Marusic, S., \& Palaniswami, M. (2014). An information framework for creating a smart city through internet of things. IEEE Internet of Things journal, 1(2), 112-121.

Atzori, L., Iera, A., Morabito, G., \& Nitti, M. (2012). The social internet of things (siot)-when social networks meet the internet of things: Concept, architecture and network 702 140,4 characterization. Computer networks, 56(16), 3594-3608.

Sicari, S., Rizzardi, A., Grieco, L. A., \& Coen-Porisini, A. (2015). Security, privacy and trust in Internet of Things: The road ahead. Computer networks, 76, 146-164.

$545 \quad 60,556$

$473 \quad 94,6$

375 125 Guinard, D., Trifa, V., Karnouskos, S., Spiess, P., \& Savio, D. (2010). Interacting with the soa-based internet of things: Discovery, query, selection, and on-demand provisioning of web services. IEEE transactions on Services Computing, 3(3), 223-235.

Yan, Z., Zhang, P., \& Vasilakos, A. V. (2014). A survey on trust management for Internet of Things. Journal of network and computer applications, 42, 120-134.

Sheng, Z., Yang, S., Yu, Y., Vasilakos, A. V., McCann, J. A., \& Leung, K. K. (2013). A survey on the ietf protocol suite for the internet of things: Standards, challenges, and opportunities. IEEE Wireless Communications, 20(6), 91-98.

Whitmore, A., Agarwal, A., \& Da Xu, L. (2015). The Internet of Things. A survey of topics and trends. Information Systems Frontiers, 17(2), 261-274.

Li, S., Da Xu, L., \& Zhao, S. (2015). The internet of things: a survey. Information Systems Frontiers, 17(2), 243-259.

352 88

328 65,6

$368 \quad 46$

88

65,6
46,286 312 78 Fonte: Dados da Pesquisa

Destaca-se a partir da Tabela 4 que dentre os 20 artigos mais citados, ou seja, os mais representativos os quais apresentaram acima de 270 citações. $\mathrm{O}$ artigo com maior destaque foi o The internet of things: A survey, escrito em 2010 por Atzori, Iera e Morabito, que 
apresentou 4.101 citações na plataforma Scopus, ou seja, representou uma diferença de $36,97 \%$ a mais em comparação com o segundo artigo mais citado quando o tema refere-se à IoT.

A partir dos documentos encontrados e por meio da nuvem de palavras (Figura 4) destacam-se os 10 termos e as respectivas vezes que cada um destes fora mencionado no campo "palavras-chave" apresentando, assim, maiores destaques nos artigos pesquisados, como por exemplo: Network (207); Wireless Sensor Networks (198); IoT (186); Systems (144); System (143); Management (141); Challenges (139); Design (113); Security (109); e Model (108).

\section{Figura 4 - Palavras-Chave Mais Relevantes}

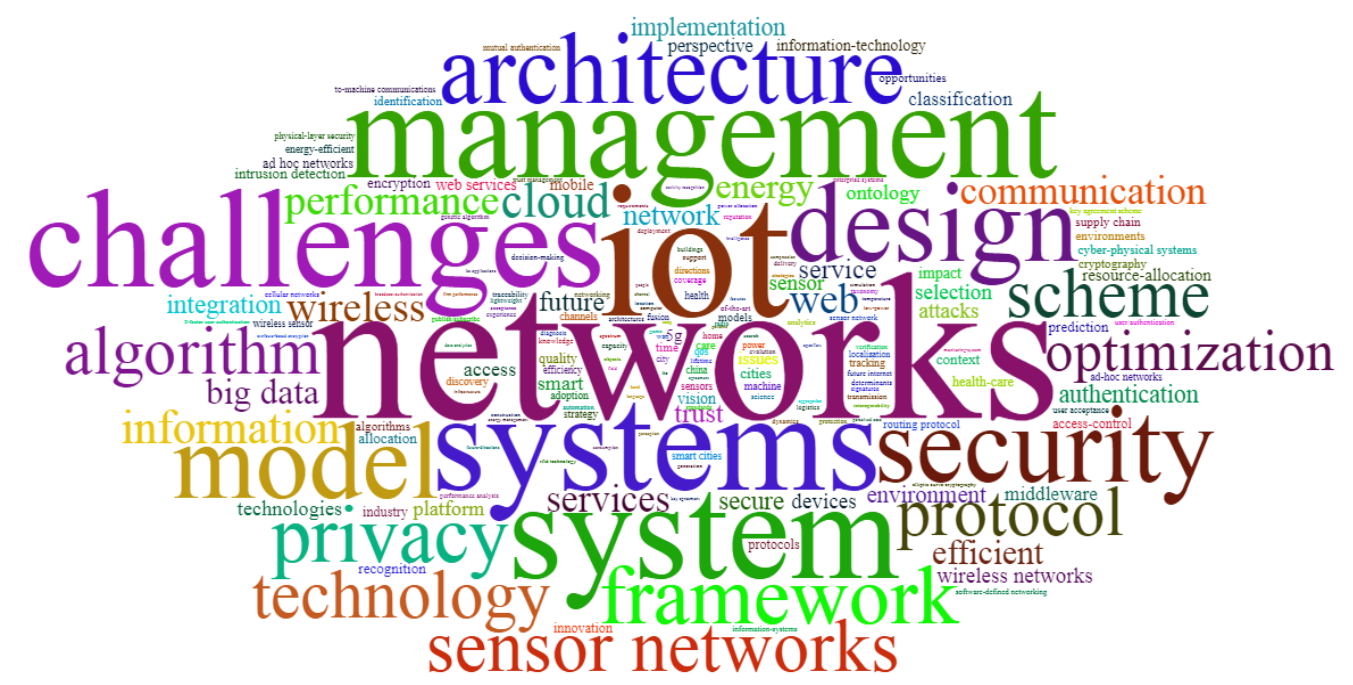

Fonte: Dados da pesquisa.

Dentre as 10 palavras-chave mencionadas e que contemplaram a nuvem de palavras (Figura 4), salienta-se que o primeiro termo citado, quando o tema se refere à IoT, faz menção a palavra network que conforme Fleisch (2010) torna-se um aparato relevante para a área de negócios em virtude de esta representar uma estrutura de rede a qual forma-se a partir da interação e comunicação entre as pessoas. Tal argumento permeia também a segunda palavra mais citada, ou seja, wireless sensor networks que compreende redes de sensores sem fio, simbolizando a existência de um transporte de energia necessária para efetivação da comunicação (FLEISCH, 2010).

Com relação à terceira palavra mais citada e a qual fora o cerne do estudo, ou seja, a IoT (Internet das Coisas), pode-se citá-lo como um termo emergente, cuja ênfase segundo Atzori, Ieda e Morabito (2010) perpassa por usuários corporativos encontrando-se em campos como a logística, automação e, por conseguinte, a área de negócios. Ademais, a quarta e 
quinta palavras encontradas, ou seja, systems e system, respectivamente, apresentaram citações significativas nos documentos, o que ratifica a conexão do termo sistema atrelado não somente à IoT como também à área de negócios, pois segundo Bonomi, et al. (2012) torna-se imprescindível o uso de sistemas por meio de dispositivos que auxiliem a mobilidade das informações.

No que tange à sexta palavra mais citada, isto é, management (gestão/gerenciamento) pode-se mencionar que de acordo com Lu, Papagiannidis e Alamanos (2018) tal termo representa a gestão de um grande número de dados, os quais formam um aparato organizado para que possa existir um controle dos mesmos. Para tanto, ao abordar a respeito da palavra challenges (desafios, sétima palavra mais citada) se aduz que tais desafios atrelados à área de negócios dizem respeito a: estabelecimento de padrões que corroborem com o progresso dos negócios, eficiência nas operações, bem como as barreiras com relação à implementação de redes mais robustas nas organizações (CHEN, 2017).

Em sequência, as últimas três palavras mais citadas, ou seja, design (desenho), security (segurança) e model (modelo), pode-se expor que tais elementos condizem para a existência de proteção da propriedade, argumento este que corrobora com o exposto por Botta, Persico e Pescapé (2016), pois servem para auxiliar tanto no processamento quanto no armazenamento das informações, assim como a integração das mesmas de forma segura.

\section{CONSIDERAÇÕES FINAIS}

Este estudo aspirou analisar as publicações sobre a IoT no universo dos negócios, mediante a ampliação dos filtros de áreas considerados por Lu et al. (2018), como finanças, pesquisa operacional, métodos quantitativos aplicados às ciências sociais, administração pública e outras. Nesse sentido, foram encontrados 2.686 documentos que versam sobre o tema (IoT), sendo 2.632 artigos, um capítulo de livro e 53 artigos que evidenciaram IoT nos procedimentos metodológicos.

Destaca-se nos achados dessa pesquisa a crescente evolução de publicações a partir de 2009 a respeito do assunto, esse viés é confirmado pelos estudos de Erfanmanesh e Abrizah (2018) e Galegale et al. (2016). Diante do exposto, foi possível verificar a tendência desses estudos voltados para área tecnologia, fator esse também evidenciado na pesquisa de Whitmore, Agarwal e Xu (2015).

Os achados corroboram com os argumentos de Erfanmanesh e Abrizah (2018) sobre o local onde ocorrera o maior número de artigos: em IEEE ACCESS The Multidisciplinary 
Open Access Journal, que de igual forma reitera a pesquisa. Desse modo, mostra-se o interesse que os estudiosos e periódicos têm dado ao tema, aumento da produtividade, criando novas estratégias de produção científica e permitindo um conhecimento mais ampliado com relação ao mercado (CHEN, 2017).

Acerca dos autores mais prolíferos sobre o tema IoT, evidencia-se a predominância dos autores asiáticos, assim denotando uma nova perspectiva, uma vez que Erfanmanesh e Abrizah (2018) localizaram a maioria como sendo provenientes de outros lugares, com destaque para Europa e Estados Unidos. Nesta perspectiva, a proporção de artigos por autor foi adequada sob a ótica dos pressupostos de Lotka (1926). Não obstante, a maioria das universidades com maior representatividade encontram-se na Ásia, sendo parte explicada pela estreita relação com os autores profícuos.

Ademais, notou-se um número significativo de autores de diversos países que abordam acerca da IoT, porém, mesmo o Brasil estando inserido nos países que versam sobre o tema, percebe-se que ainda são incipientes os estudos em comparação com os países asiáticos. Adiciona-se também que conforme as palavras-chave encontradas nos artigos observa-se uma convergência entre a IoT com a área de negócios, ou seja, a primeira vem a auxiliar a segunda, no sentido de promover a troca de dados e informações, confidenciáveis ou não, de forma fidedigna e com tempestividade.

A presente investigação contribui sob dois vieses. O primeiro consiste na ampliação do escopo e período das análises anteriores, que versassem sobre revisões bibliométricas e/ou análises de produções científicas envolvendo o tema IoT no campo dos negócios. A segunda concerne à apresentação e aos demonstrativos de utilização do package Bibliometrix no software R, no intuito de auxiliar pesquisadores a realizarem estudos metodologicamente semelhantes a este, visando maior praticidade e dinamismo ao trabalhar com big data (grande quantidade de informações).

Sugere-se para posteriores estudos que o tema em questão (IoT) seja analisado por meio de outras plataformas de dados, no intuito de visualizar a evolução de tal tecnologia. Além disso, estudos que compreendam a aplicação da IoT em outros campos de conhecimento também emergem como forma sugestiva.

\section{REFERÊNCIAS}

ARIA, M.; CUCCURULLO, C. bibliometrix: An R-tool for comprehensive science mapping analysis. Journal of Informetrics, v. 11, n. 4, p. 959-975, 2017. 
ATZORI, L.; IERA, A.; MORABITO, G. The internet of things: A survey. Computer networks, v. 54, n. 15 , p. 2787-2805, 2010.

BALDINI, G.; BOTTERMAN, M.; NEISSE, R.; TALLACCHINI, M. Ethical design in the internet of things. Science and engineering ethics, v.24, n. 3, p. 905-925, 2018.

BONOMI, F.; MILITO, R.; ZHU, J.; ADDEPALLI, S. Fog computing and its role in the internet of things. In Proceedings of the first edition of the MCC workshop on Mobile cloud computing, p. 13-16, 2012.

BOTTA, A.; DE DONATO, W.; PERSICO, V.; PESCAPÉ, A. Integration of cloud computing and internet of things: a survey. Future generation computer systems, v. 56, p. 684-700, 2016.

CHEN, Y. Integrated and Intelligent Manufacturing: Perspectives and Enablers. Engeneering, v. 3, n. 5, p. 588-595, 2017.

DIAS, R. R. de F. Internet das coisas sem mistérios: uma nova inteligência para os negócios. São Paulo: Netpress Books, 2016.

ERFANMANESH, M.; ABRIZAH, A. Mapping worldwide research on the Internet of Things during 2011-2016. The Electronic Library, v. 36, n. 6, p. 979-992, 2018.

FLEISCH, E. What is the internet of things? An economic perspective. Economics, Management \& Financial Markets, v. 5, n. 2, p. 1-27, 2010.

GAleGAle, G. P.; SIQUEIRA, É.; SILVA, C. B. H E.; SOUZA, C. A. de. Internet das Coisas aplicada a negócios - Um estudo bibliométrico. JISTEM - Journal of Information Systems and Technology Management, v. 13, n. 3, p. 423-438, 2016.

GUBBI, J et al. Internet of Things (IoT): A vision, architectural elements, and future directions. Future generation computer systems, v. 29, n. 7, p. 1645-1660, 2013.

IANSITI, M.; LAKHANI, K. R. Digital ubiquity: how connections, sensors and data are revolutionizing business. Harvard Business Review, v. 92, n.11, p. 90-99, 2014.

LEE, I.; LEE, K. The Internet of Things (IoT): Applications, investments, and challenges for enterprises. Business Horizons, v. 58, n. 4, p. 431-440, 2015.

LOTKA, A. J. Elements of physical biology. Science Progress in the Twentieth Century (1919-1933), v. 21, n. 82, p. 341-343, 1926.

LU, Y.; PAPAGIANNIDIS, S.; ALAMANOS, E. Internet of Things: A systematic review of the business literature from the user and organisational perspectives. Technological Forecasting and Social Change, v. 136, p. 285-297, 2018.

KOSMATOS, E. A.; TSELIKAS, N. D.; BOUCOUVALAS, A. C. Integrating RFIDs and smart objects into a unified internet of things architecture. Advances in Internet of Things, v. 1, n. 1, p. 5-12, 2011. 
MAN, L. C. K.; NA, C. M.; KIT, N. C. IoT-based asset management system for healthcarerelated industries. International Journal of Engineering Business Management, v. 7, n. Godište 2015, p. 7-19, 2015.

MARTINS, G. A.; THEÓPHILO, C. R. Metodologia da Investigação Científica para Ciências Sociais Aplicadas. São Paulo: Atlas, 2009.

MEDEIROS, F. S. B.; COLPO, I.; SCHNEIDER, V. A.; CARVALHO, P. S. Internet of Things (IoT): Uma investigação do Conhecimento Científico em Artigos Acadêmicos na Última Década. Revista Eletrônica de administração e Turismo, v. 12, n. 7, p. 1652-1674, 2018.

MISHRA, D.; GUNASEKARAN, A.; CHILDE, S.J.; PAPADOPOULOS, T.; DUBEY, R.; WAMBA, S. Vision, applications and future challenges of internet of things: a bibliometric study of the recent literature. Industrial Management and Data Systems, v. 116, n. 7, p. 1331-1355, 2016.

PACHECO, F. B.; KLEIN, A. Z.; RIGHI, R. R. Modelos de negócio para produtos e serviços baseados em internet das coisas: uma revisão da literatura e oportunidade de pesquisas futuras. REGE - Revista de Gestão, v. 23, n. 1, p. 41-51, 2016.

PORTER, M, E.; HEPPELMANN, J, E. How smart, connected products are transforming competition. Harvard business review, v. 92, n. 11, p. 64-88, 2014.

OBERLÄNDER, A. M.; RÖGLINGER, M.; ROSEMANN, M.; KEES, A. Conceptualizing business-to-thing interactions-A sociomaterial perspective on the Internet of Things. European Journal of Information Systems, v. 27, n. 4, p. 486-502, 2018.

SHIN, D. A socio-technical framework for internet-of-things design: a human-centered design for the internet of things. Telematics and Informatics, v. 31, n. 4, p. 519-531, 2014.

SICARI, S.; RIZZARDI, A.; GRIECO, L. A.; COEN-PORISINI, A. Security, privacy and trust in Internet of Things: The road ahead. Computer networks, v. 76, p. 146-164, 2015.

STANKOVIC, J. A. Research directions for the internet of things. IEEE Internet of Things Journal, v. 1, n. 1, p. 3-9, 2014.

VAN DEURSEN, A. J. A. M.; MOSSBERGER, K. Any thing for anyone? A new digital divide in Internet-of-things skills. Policy and Internet, v. 10, n. 2, p. 122-140, 2018.

WHITMORE, A.; AGARWAL, A.; XU, L. D. The Internet of Things - A survey of topics and trends. Information Systems Frontiers, v. 17, n. 2, p. 261-274, 2015.

XU, X. Internet of things in service innovation. Amfiteatru Economic Journal, v. 14, (Special n. 6), p. 698-719, 2012.

YANG, L.; YANG, S. H.; PLOTNICK, L. How the internet of things technology enhances emergency response operations. Technological Forecasting and Social Change, v. 80, n. 9, p. 1854-1867, 2013. 
ZANELLA, A.; BUI, N.; CASTELLANI, A.; VANGELISTA, L.; ZORZI, M. Internet of things for smart cities. IEEE Internet of Things journal, v. 1, n. 1, p. 22-32, 2014.

\section{Como Referenciar este Artigo, conforme ABNT:}

FRARE, A. B; MARTINS, A. S. R; FERNANDES, C. M. G; HORZ, V; QUINTANA, A. C; Internet das Coisas Aplicada aos Negócios: Análise das Publicações Utilizando o Bibliometrix. Rev. FSA, Teresina, v.18, n. 7, art. 4, p. 55-76, jul. 2021.

\begin{tabular}{|l|c|c|c|c|c|}
\hline \multicolumn{1}{|c|}{ Contribuição dos Autores } & $\begin{array}{c}\text { A. B. } \\
\text { Frare }\end{array}$ & $\begin{array}{c}\text { A. S. R. } \\
\text { Martins }\end{array}$ & $\begin{array}{c}\text { C. M. G. } \\
\text { Fernandes }\end{array}$ & V. Horz & $\begin{array}{c}\text { A. C. } \\
\text { Quintana }\end{array}$ \\
\hline 1) concepção e planejamento. & $\mathrm{X}$ & $\mathrm{X}$ & $\mathrm{X}$ & $\mathrm{X}$ & $\mathrm{X}$ \\
\hline 2) análise e interpretação dos dados. & $\mathrm{X}$ & $\mathrm{X}$ & $\mathrm{X}$ & $\mathrm{X}$ & $\mathrm{X}$ \\
\hline 3) elaboração do rascunho ou na revisão crítica do conteúdo. & $\mathrm{X}$ & $\mathrm{X}$ & $\mathrm{X}$ & $\mathrm{X}$ & $\mathrm{X}$ \\
\hline 4) participação na aprovação da versão final do manuscrito. & $\mathrm{X}$ & $\mathrm{X}$ & $\mathrm{X}$ & $\mathrm{X}$ & $\mathrm{X}$ \\
\hline
\end{tabular}

